\title{
ICMM guidance and resources for integrating closure into business decision making processes
}

\author{
D Brock International Council on Mining and Metals, United Kingdom
}

\begin{abstract}
The International Council on Mining and Metals (ICMM) is an international organisation dedicated to a safe, fair and sustainable mining and metals industry. Bringing together 27 mining and metals companies and over 35 regional and commodities associations, it strengthens environmental and social performance and serves as a catalyst for change, enhancing mining's contribution to society.

Mine closure has become one of the most challenging issues facing mining companies, communities and governments around the world. For mining companies, safety, environmental and social risks can occur, and significant financial provision will be required to manage and address these risks. Moreover, planned mine closures around the world are expected to increase over the next decade, offering a unique opportunity to improve closure-related performance.

Integrated mine closure is a dynamic and iterative process that takes into account environmental, social and economic considerations at an early stage of a mine's development. Fundamental to this process is the need to consider closure as an integral part of the mine operations' core business.

The ICMM Integrated Mine Closure: Good Practice Guide (2nd edition, 2019) and supporting resources assist in this process, providing guidance in delivering key elements of mine closure planning and implementation. These resources have been developed by the ICMM Closure Working Group, made up of mine closure practitioners from leading global mining companies. Input was also sought from other key disciplines as well as from industry specialists, academia and government representatives. Resources developed to support companies in implementing the key concepts of the ICMM Closure Guide include:

1) Financial Concepts for Mine Closure (2019) - for use by ICMM members and the wider industry to communicate and enhance the understanding of the various types of closure cost estimates as they relate to mine closure, enabling consistent understanding and communication across the industry, between industry disciplines and with external stakeholders.
\end{abstract}

2) Key Performance Indicators: Tool for Closure (2020) - designed to help companies develop contextrelevant performance indicators that drive accountability and performance. This tool provides a set of Key Performance Areas and illustrative examples of Key Performance Indicators to support implementation of good closure practices at an operational level.

3) Closure Maturity Framework (2020) a tool developed for companies to use to build a common understanding of closure concepts across an asset's life cycle and across mining disciplines. The intent of the Closure Maturity Framework is to Map, Motivate and Measure the status of assets on their journey to sustainable closure. It will drive conversation amongst industry stakeholders to converge thinking, decision making and drive positive industry change.

4) Training materials (2020) - intended to promote a more integrated outcome and control of closure planning and implementation activities at an operational level, as well as provide practical resources to assist in planning for successful mine closure.

This paper provides an overview of how the Integrated Mine Closure: Good Practice Guide and supporting resources can be used to drive improved performance and integration of closure into business decision making processes to improve closure performance and reduce closure liabilities.

Keywords: closure, integration, guidance 


\section{Introduction}

The International Council on Mining and Metals (ICMM) brings together 27 mining and metal companies and over 35 regional and commodities associations to strengthen environmental and social performance and enhance mining's contribution to society. To support this, every ICMM company member adheres to ICMM's Mining Principles which incorporates comprehensive environmental, social and governance requirements, robust site-level validation of performance expectations and credible assurance of corporate sustainability reports. The vision of ICMM is for mining and metals to be a respected industry, trusted to operate responsibly and contribute to sustainable development.

Closure and legacy issues are a continuing source of material risk to ICMM company members and reputational risk for the industry. Planned mine closures around the world are expected to increase over the next decade offering a unique opportunity to improve closure-related performance.

As part of ICMM's Mining Principles, our members commit to plan and design for closure in consultation with relevant authorities and stakeholders, implement measures to address closure-related environmental and social aspects, and make financial provision to enable agreed closure and post-closure commitments to be realised (ICMM, 2019b). To support our members and the wider industry in doing this, ICMM published its Integrated Mine Closure: Good Practice Guide (Closure Guide) to assist companies in planning for effective, integrated and responsible mine closure.

The Closure Guide is premised on the concept of integration which is a dynamic and iterative process that takes into account environmental, social and economic considerations at an early stage of mine development and is developed throughout the life of the mine. Fundamental to this is the need to consider closure as an integral part of the mine operations' core business.

To operationalise the key concepts of the Closure Guide, ICMM has produced supplementary resources to support in building capacity at the asset level and facilitate the integration of closure into key site functions and core business practices. These resources include the Financial Concepts for Mine Closure information document, Key Performance Indicators: Tool for Closure, Closure Maturity Framework and the Closure Guide Training Materials.

This paper provides a brief overview of the Closure Guide and supporting resources developed to support the industry in planning for and implementing closure by means of an integrated approach.

\section{Development}

The ICMM Closure Working Group, which is made up of mine closure practitioners from leading global mining companies, recognised the considerable evolution in mine closure practices in the last decade, and that guidance was required to better reflect a modern approach. An important aspect of this was the need to emphasize the importance of integrating closure into business planning and decision-making processes in order to reduce potential risks and liabilities.

This led to the development of the Integrated Mine Closure: Good Practice Guide which draws on existing good practice examples and publications on mine closure available across the globe. To support the integration of the Closure Guide and implementation at the operational level, supplementary resources were developed by the Closure Working Group. These included the Financial Concepts for Mine Closure, Key Performance Indicators: Tool for Closure, Closure Maturity Framework and Closure Guide Training Materials.

Meetings of the Working Group provided opportunities to discuss the content and agree on the structure of the resources developed. Once the draft documents were in place, these were tested with company representatives specialising in water, finance, community, environment and other key disciplines as required. 


\section{$3 \quad$ Resources}

Integrated Mine Closure: Good Practice Guide (2nd Edition, 2019)

Designing for closure means integrating closure activities into the mine business plan. This includes the short, medium and Life of Mine (LOM) planning processes throughout the mine life taking into account environmental, social and economic considerations (ICMM, 2019a).

As with closure planning itself, integrating closure into the LoM plan is an iterative process that incorporates developing information and experience over the mining life cycle. The earlier this approach is followed in the life of a mine, the greater the opportunity for proactive planning. This provides a consistent approach over the life cycle of projects for reporting and management of long-term liabilities in ensuring a positive social and environmental legacy is left behind post-closure (ICMM, 2019a).

The Closure Guide is intended to promote a disciplined approach to integrated closure planning and increase the uniformity of good practice across the sector. A pathway that is aligned with the organisation of the Closure Guide can be drawn through the key elements of mine closure (Figure 1).

The Closure Guide uses a risk and opportunity-based process to guide the practitioner through the iterative process of planning for final and progressive closure in a considered manner, as well as tactics for considering sudden or temporary closure.

The Closure Guide also includes supplementary information in the form of a series of tools to aid the practitioner in developing closure plans and carrying out activities in support of closure. The relevant tools are listed throughout the document. Case studies are drawn from the experiences of both ICMM member companies and other mines and are presented throughout the document to illustrate recent, practical experience with mine closure at sites around the world.

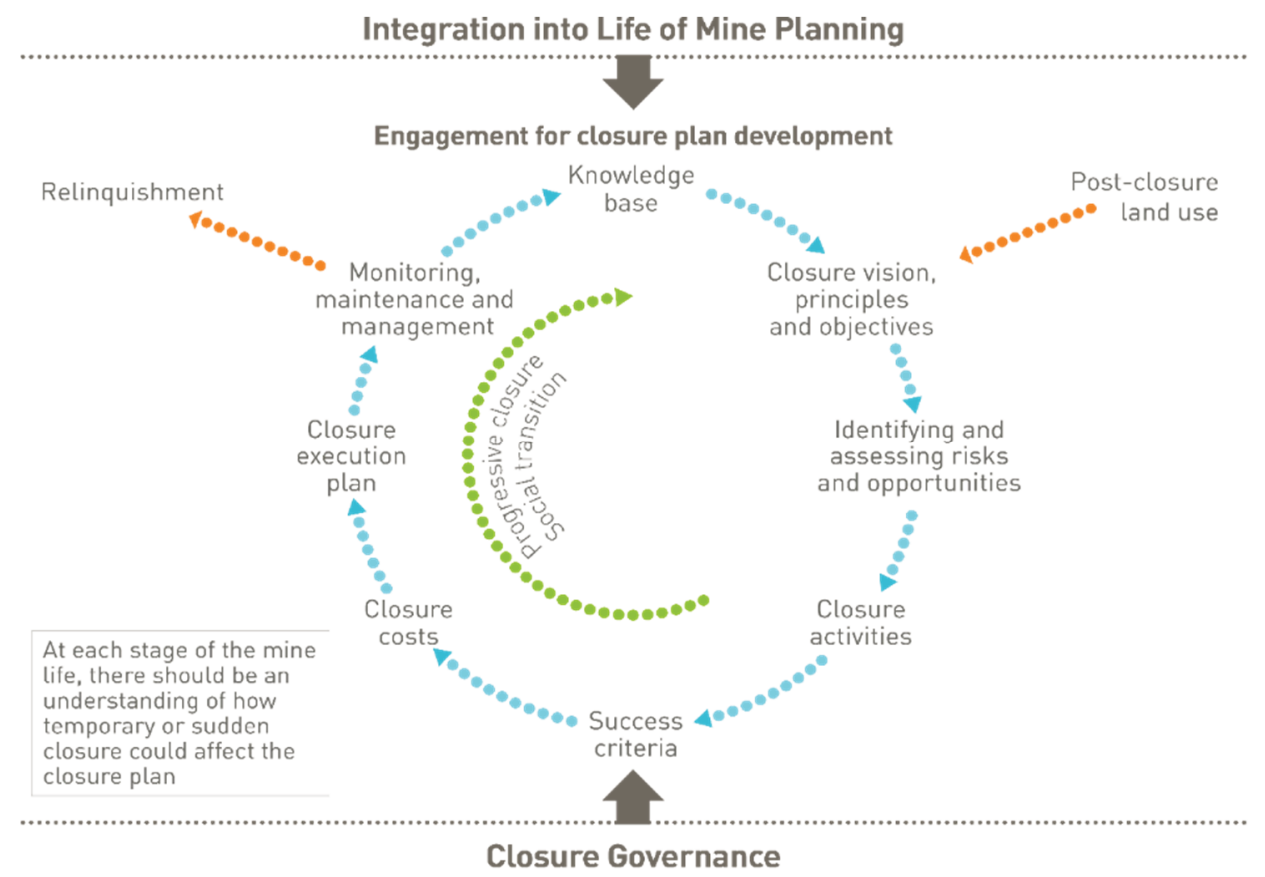

Figure 1 Key elements of mine closure planning and implementation (ICMM, 2019a)

\section{Financial Concepts for Mine Closure (2019)}

ICMM members commit to making financial provision to address the environmental and social aspects of closure (ICMM, 2019b). To support this and to enhance the understanding of key financial concepts as they relate to mine closure, ICMM has developed the Financial Concepts for Mine Closure guidance. 
Several types of cost estimates are used to characterise the financial aspects of closure and to understand the risks and opportunities for preparing for closure. An understanding of these approaches vary, consistent communication and engagement within companies and with external stakeholders is challenging and limits the industry's ability to benchmark performance. It is important for mining companies to make clear distinctions between the different types of cost estimates as they serve different purposes. The Financial Concepts for Mine Closure guidance is intended to provide general conceptual guidance across a wide range of factual circumstances. The document defines and contextualises the key concepts related to closure costing, accounting and reporting requirements and the purpose of each.

The document is mainly structured around the basic types of closure cost estimates as outlined in Figure 2, providing an overview of the cost types as well as general inclusions, exclusions and considerations.
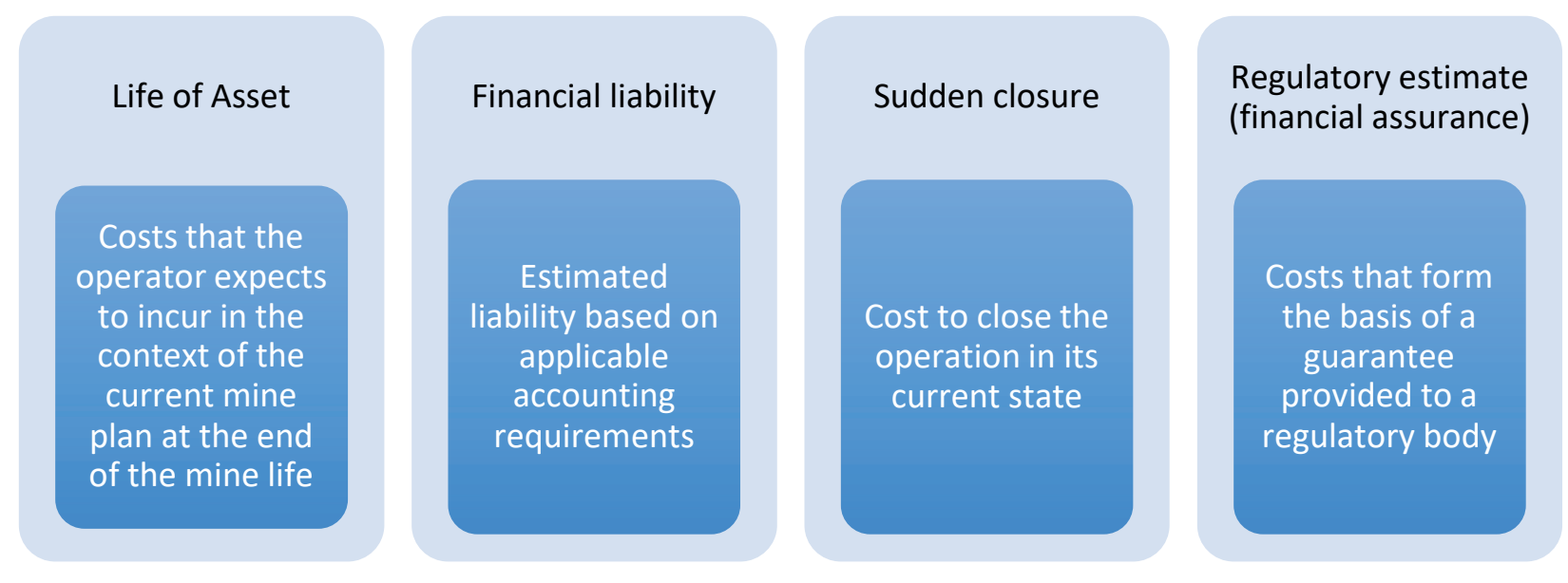

Figure 2 Closure cost estimate types (ICMM 2019c)

\section{Key Performance Indicators: Tool for Closure (2020)}

An operation with clear closure objectives and related context-relevant indicators, against which performance can be measured, are best placed to achieve their closure objectives.

The Key Performance Indicators: Tool for Closure provides a set of Key Performance Areas (KPA) and illustrative examples of Key Performance Indicators (KPIs) to support implementation of good closure practices at an operational level throughout the mine life cycle.

This tool does not prescribe what indicators should be, but instead provides illustrative examples for asset leaders, including application notes to help mining companies develop their own KPIs to track their internal performance on closure management. These site-level measures may be aggregated throughout the organisation to provide visibility at key decision-making points, centrally and at senior management level. Some suggestions for aggregated indicators are also provided in the tool (ICMM, 2020a).

The KPI Tool is based on a management system approach around the 'plan - do - check - act' cycle. As the mine evolves, the KPIs will likely change to reflect the evolving closure vision and objectives (ICMM, 2020a).

\section{Closure Maturity Framework (2020)}

The Closure Maturity Framework is designed to help companies map their current position, motivate improvement, and measure the state of their operations' progress towards sustainable closure. It outlines a pathway towards achieving desired post-closure land uses and closure vision while considering relevant aspects of mine design, operations, and technology (ICMM, 2020b). The tool will assist them in understanding what future work is needed to achieve their closure objectives with the goal of driving continuous improvement in closure performance. 
The Closure Maturity Framework tool has two components 1) a User Guide that describes each of the maturity levels and helps companies categorise the status of their sites and 2) an interactive Excel Tool that helps companies assess closure maturity at the site level. It can serve as a communication tool that drives conversation between different business units and levels of a company to converge thinking and align decision making. It can also facilitate the aggregation of results from multiple sites to enable companies to understand closure performance within a portfolio.

As the approach taken for closure will vary depending on the Life of Asset (LoA) and time remaining before predicted closure, criteria have been developed for the five main asset stages (exploration, early operations, mature operations, pre closure and closure execution and post closure monitoring). The criteria for each phase of the asset life cycle are based on the key elements presented in the Closure Guide and are based on maturity levels ranging from nascent to leading practice which need to be achieved before moving to the next maturity level.

Assets can record their current maturity level and aspirational maturity level, with the output recorded on visual diagrams to aid communication and understanding between teams and business units. The aim is to drive and enable honest conversations on how to move nearer their aspirational level of maturity.

The Closure Maturity Framework is best suited for assets in the earlier phases of development, where incorporation of closure into decision making and planning is possible. It becomes less useful for sites approaching closure imminently or already closed sites, which have not followed the integrated closure approach (ICMM, 2020b).

\section{Training Materials (2020)}

To encourage implementation of the Closure Guide, training materials have been developed to support conversations of mine closure challenges, opportunities and global good practice implementation at the operational level.

Presented in a slide pack format, the training materials have been developed specifically for site closure specialists who are familiar with the closure activities of the site which need to be delivered. The target audience are site-based staff with a role in closure planning and delivery who are not themselves closure specialists. Additionally, to add greater applicability, these materials can be customised to an individual site or company.

The training materials are based on the key elements of the Closure Guide and are in five slide deck modules with accompanying case studies and practical exercises. The slide decks have embedded facilitator notes, providing suggestion on how to structure the modules and training to focus on specific elements and considerations for mine closure. Each module contains learning aims and objectives, which includes theory and practical exercises to encourage the audience to think about their current site-specific closure opportunities or challenges. The training materials also include activities using ICMM's Closure Maturity Framework to help site teams communicate and understand their current and aspirational level of closure maturity (ICMM, 2020c).

\section{Conclusion}

The Closure Guide and supporting resources provides ICMM members and other responsible mining companies with guidance needed to effectively integrate closure across the mining lifecycle. This in turn creates the opportunity for companies to be proactive in identifying and addressing risks early before they become material with the potential to compromise eventual mine closure.

Mining companies must prioritise responsible closure of their mines to ensure that the social license of mining is not diminished and continues to deliver the raw materials required for ongoing sustainable development of society. 


\section{Acknowledgement}

The authors would like to thank the ICMM Closure Working Group, ICMM members and team, as well as other independent reviewers who contributed by providing direction, input and expert review to the development of the resources.

\section{References}

International Council on Mining and Metals ICMM, 2019a, Integrated Mine Closure: Good Practice Guide, 2nd ed, London.

International Council on Mining and Metals ICMM, 2019b, 6 Environmental Performance, viewed 19 February 2020, https://www.icmm.com/mining-principles/6

International Council on Mining and Metals ICMM, 2019c, Financial Concepts for Mine Closure, London.

International Council on Mining and Metals ICMM, 2020a, Key Performance Indicators: Tool for Closure, London.

International Council on Mining and Metals ICMM, 2020b, Closure Maturity Framework (2020), viewed 22 February 2020, https://www.icmm.com/en-gb/guidance/environmental-stewardship/closure-maturity-framework

International Council on Mining and Metals ICMM, 2020c, Adapting ICMM's 'Integrated Mine Closure: Good practice guide' into

training materials (2020), viewed 22 February 2020, https://www.icmm.com/en-gb/guidance/environmentalstewardship/integrated-mine-closure-training 